\title{
Automated three-dimensional tracking of the left ventricular myocardium in time-resolved and dose-modulated cardiac CT images using deformable image registration
}

Vikas Gupta, Jonas Lantz, Lilian Henriksson, Jan Engvall, Matts Karlsson, Anders Persson and Tino Ebbers

The self-archived postprint version of this journal article is available at Linköping University Institutional Repository (DiVA):

http://urn.kb.se/resolve?urn=urn:nbn:se:liu:diva-147433

N.B.: When citing this work, cite the original publication.

Gupta, V., Lantz, J., Henriksson, L., Engvall, J., Karlsson, M., Persson, A., Ebbers, T., (2018), Automated three-dimensional tracking of the left ventricular myocardium in time-resolved and dosemodulated cardiac CT images using deformable image registration, Journal of Cardiovascular Computed Tomography, 12(2), 139-148. https://doi.org/10.1016/j.jcct.2018.01.005

Original publication available at:

https://doi.org/10.1016/j.jcct.2018.01.005

Copyright: Elsevier

http://www.elsevier.com/

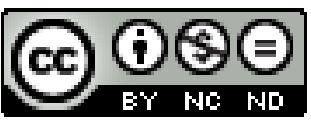




\title{
Automated three-dimensional tracking of the left ventricular myocardium in time-resolved and dose-modulated cardiac CT images using deformable image registration
}

\author{
Vikas Gupta, $\mathrm{PhD}{ }^{\star a, b}$, Jonas Lantz, $\mathrm{PhD}{ }^{\mathrm{a}, \mathrm{b}}$, Lilian Henriksson, $\mathrm{MSc}^{\mathrm{b}, \mathrm{c}}$, Jan Engvall, MD, $\mathrm{PhD}{ }^{\mathrm{a}, \mathrm{b}}$, Anders Persson, MD, \\ $\mathrm{PhD}{ }^{\mathrm{b}, \mathrm{c}}$, Matts Karlsson, $\mathrm{PhD}^{\mathrm{b}, \mathrm{d}}$, Tino Ebbers, $\mathrm{PhD}^{\mathrm{a}, \mathrm{b}}$ \\ ${ }^{a}$ Division of Cardiovascular Medicine, Department of Medical and Health Sciences, Linköping University, Linköping, Sweden \\ ${ }^{b}$ Center for Medical Image Science and Visualization (CMIV), Linköping University, Department of Medical and Health Sciences, Linköping, Sweden \\ ${ }^{c}$ Department of Radiology and Department of Medical and Health Sciences, Linköping University, Linköping, Sweden \\ ${ }^{d}$ Division of Applied Thermodynamics and Fluid Mechanics, Department of Management and Engineering, Linköping University, Linköping, Sweden
}

Background: Assessment of myocardial deformation from time-resolved cardiac computed tomography (4D CT) would augment the already available functional information from such an examination without incurring any additional costs. A deformable image registration (DIR) based approach is proposed to allow fast and automatic myocardial tracking in clinical 4D CT images.

Methods: Left ventricular myocardial tissue displacement through a cardiac cycle was tracked using a B-spline transformation based DIR. Gradient of such displacements allowed Lagrangian strain estimation with respect to end-diastole in clinical 4D CT data from ten subjects with suspected coronary artery disease. Dice similarity coefficient (DSC), point-to-curve error (PTC), and tracking error were used to assess the tracking accuracy. Wilcoxon signed rank test provided significance of tracking errors. Topology preservation was verified using Jacobian of the deformation. Reliability of estimated strains and torsion (normalized twist angle) was tested in subjects with normal function by comparing them with normal strain in the literature.

Results: Comparison with manual tracking showed high accuracy (DSC: $0.99 \pm 0.05$; PTC: $0.56 \mathrm{~mm} \pm 0.47 \mathrm{~mm}$ ) and resulted in determinant (Jacobian) $>0$ for all subjects, indicating preservation of topology. Average radial $(0.13 \mathrm{~mm})$, angular (0.64) and longitudinal $(0.10 \mathrm{~mm})$ tracking errors for the entire cohort were not significant $(p>0.9)$. For patients with normal function, average strain [circumferential, radial, longitudinal] and peak torsion estimates were: [-23.5\%, $31.1 \%,-17.2 \%]$ and $7.22^{\circ}$, respectively. These estimates were in conformity with the reported normal ranges in the existing literature.

Conclusions: Accurate wall deformation tracking and subsequent strain estimation are feasible with the proposed method using only routine time-resolved 3D cardiac CT.

Key words: Cardiac Computed Tomography, 4D CT, image registration, strain analysis, myocardial deformation, torsion

\section{Introduction}

Quantification of myocardial deformation from timeresolved three-dimensional cardiac CT (or 4D CT) would extend the amount of functional information that is currently obtained from such an examination. 4D CT has several advantages over other imaging techniques including a high spatial resolution, short scan time and, often, fewer contraindications. Obtaining multiple functional parameters from these data would extend the usability of 4D CT further. Myocardial strain, that provides an intuitive and orientation invariant estimate of wall deformations, can be computed from 4D CT without incurring any additional acquisition $\operatorname{cost}^{1+3}$. Especially, in patients with known or suspected coronary artery disease (CAD), myocardial deformation assessment would be an excellent complement to the detection of coronary artery stenosis.
Strain is typically obtained after tracking the myocardium over an entire cardiac cycle. Unfortunately, 4D CT images often have low contrast in the myocardial wall that makes its tracking extremely challenging in a large part of the cardiac cycle. Few methods exist to perform this tracking. A multi-channel Diffeomorphic Demons based nonlinear spatiotemporal framework was used in Peyrat et $\mathrm{al}^{4}$ to obtain trajectories of physical points in both patient and synthetic data. In another image registration based approach ${ }^{56}$, enforced displacements from B-spline deformable model served as boundary conditions to a biomechanical model in data from ten canines. Biomechanical modeling was also used ${ }^{11}$, wherein a left ventricular mesh model enabled the wall deformation tracking in different phases of a cardiac cycle ${ }^{11}$. In a different approach ${ }^{2}$, threedimensional (3D) motion tracking was performed by constraining intensity constancy, myocardial volume changes 
and motion smoothness assumptions. While these studies have shown promising results, they are limited by high accuracy errors $>2.9 \mathrm{~mm}^{2}$ or long processing time of up to 900 minutes $^{4}$, or evaluation in only synthetic and canine data ${ }^{45}$, which hampers their advancement to clinical use.

The purpose of this study was to test the clinical feasibility of a fast, accurate and automatic deformable image registration based myocardial tracking in routine 4D CT examinations and to show its potential for reliable strain estimations.

\section{Materials and Methods}

\subsection{Study population}

This retrospective study was approved by the Regional Ethical Review Board and complied with the Helsinki Declaration. Ten patients (Table 1 with suspected CAD were included. Informed consent was obtained from all subjects.

\subsection{Image acquisition}

Retrospectively-gated 4D CT images were acquired for referred clinical investigations using a dual source CT scanner (SOMATOM Force, Siemens, Germany) with automated exposure control (CARE Dose4D) and tube voltage selection (CARE kV). ECG-triggered dose modulation reduced the mean radiation dose by up to $30-50 \% 7$. Scanning parameters were: pitch 0.15-0.34 (heart rate dependent); gantry rotation time $0.25 \mathrm{~s}$; temporal resolution $66 \mathrm{~ms}$; reference kV 100; quality reference mAs 276; and detector collimation $1920.6 \mathrm{~mm}$. Data of the left ventricle were acquired over 3-4 cardiac cycles depending on the heart rate. Subsequently, cardiac volumes were reconstructed for 20 phases covering the RR-interval, resulting in a reconstructed temporal resolution of $45 \pm$ $4.6 \mathrm{~ms}$. Reconstruction algorithms included medium softtissue convolution (Bv36) and Advanced Modeled Iterative Reconstruction (Siemens) at noise reduction level 4. Thus obtained multi-phase volumes were further reformatted to short-axis views and resampled to obtain $0.5 \times 0.5 \times 0.5$ $\mathrm{mm}$ voxels.

\subsection{Myocardial segmentation}

The epi- and endocardial boundaries of the myocardium were identified automatically using a commercial tool, called Segment (Medviso AB, Lund Sweden) ${ }^{8}$, in the volume corresponding to the first phase after the ECG-trigger. This phase typically represents end-diastole that is considered to be the least stressed phase for the musculature in a cardiac cycle ${ }^{9}$. These boundaries were manually corrected by an experienced observer, if required. A threedimensional mask spanning the transmural myocardium (Figure 1) was created from these boundaries and the position of masked voxels served as the reference myocardial configuration the displacement of which was tracked over a cardiac cycle. As the tracking is performed for the entire myocardium, similar to MRI based myocardial tissue tagging and displacement encoding with stimulated echoes (DENSE), the precision of epi- and endocardial segmentation becomes less critical for the strain estimation.

\subsection{Deformable image registration (DIR)}

Temporal displacement of the transmural myocardium was tracked using a deformable image registration (DIR) approach that is based on a free-form deformation mode ${ }^{10}$ with four multi-resolution hierarchical levels of B-spline control points ${ }^{11}$. A Lagrangian framework was used, wherein, the deformations were estimated with respect to the reference configuration (Figure 1 defined at end diastole. Over a cardiac cycle, the three-dimensional displacement of the reference myocardial configuration, identified earlier (section 2.3), was then obtained by using the DIR between each three-dimensional image (moving, $I_{m_{i}}$ ) at phase, $i$, and the reference image, $I_{r_{i}}$ (Figure 2). The estimated displacement field, $\hat{T}_{i}$, was obtained by maximizing a similarity measure, $\phi$, as described in the following equation ${ }^{12}$ :

$$
\hat{T}_{i}=\underset{T_{i}}{\arg \max } \phi\left(T_{i}\left(I_{m_{i}}\right), I_{r}\right),
$$

where, $T_{i}$ is the iterative transformation applied to the moving image, $I_{m_{i}}$, to match the reference image, $I_{r}$. A fast adaptive stochastic gradient descent (FASGD) strategy $\frac{13}{13}$ was used to iteratively update the transformation, $T_{i}$. FASGD performs computationally efficient random data sampling for gradient calculation at each iteration of gradient descent optimization and allows unsupervised setting of adaptive step sizes. Normalized Mutual information $(\mathrm{NMI})^{14}$, computed using equation 2 , served as the similarity measure to handle intensity inhomogeneities and non-stationarity caused by dose modulation and cardiac contractions, respectively.

$$
\operatorname{NMI}\left(I_{r}, I_{\hat{m}_{i}}\right)=\frac{\sum_{\hat{m}_{i} \in H_{\hat{m}_{i}}} \sum_{r \in H_{r}} p\left(r, \hat{m}_{i}\right) \log _{2}\left(p_{r}(r) p_{\hat{m}_{i}}\left(\hat{m}_{i}\right)\right)}{\sum_{\hat{m}_{i} \in H_{\hat{m}_{i}}} \sum_{r \in H_{r}} p\left(r, \hat{m}_{i}\right) \log _{2} p\left(r, \hat{m}_{i}\right)}
$$

Here, $H_{r}$ and $H_{\hat{m}_{i}}$ are sets of regularly spaced intensity bin centers; $p$ is the discrete joint probability; and $p_{r}$ and $p_{\hat{m}_{i}}$ are the marginal discrete probabilities of the reference $\left(I_{r}\right)$ and deformed moving images $\left(I_{\hat{m}_{i}}\right)$ at phase $i$ of the cardiac cycle, respectively.

\subsection{Strain and torsion estimation}

Myocardial strain was estimated by using the displacement fields resulting from the proposed DIR approach. If $(u, v, w)$ represent the displacement of a reference configuration in directions $(x, y, z)$ respectively, the deformation gradient tensor, $\boldsymbol{F}$, is defined as ${ }^{15}$ :

$$
F=I+\left(\begin{array}{lll}
\frac{\partial u}{\partial x} & \frac{\partial u}{\partial y} & \frac{\partial u}{\partial z} \\
\frac{\partial v}{\partial x} & \frac{\partial v}{\partial y} & \frac{\partial v}{\partial z} \\
\frac{\partial w}{\partial x} & \frac{\partial w}{\partial y} & \frac{\partial w}{\partial z}
\end{array}\right)
$$


Table 1: Patient Characteristics

\begin{tabular}{|c|c|c|c|c|c|c|}
\hline & \multicolumn{2}{|c|}{ All subjects $(\mathrm{N}=10)$} & \multicolumn{2}{|c|}{ Female $(\mathrm{N}=3)$} & \multicolumn{2}{|c|}{ Male $(\mathrm{N}=7)$} \\
\hline & mean \pm std. dev. & range & mean \pm std. dev. & range & mean \pm std. dev. & range \\
\hline Age [years] & $61.0 \pm 12.4$ & $36.0-74.0$ & $67.0 \pm 5.6$ & $62.0-73.0$ & $58.4 \pm 14.0$ & $36.0-74.0$ \\
\hline Height $[\mathrm{cm}]$ & $173.8 \pm 6.7$ & $162.5-187.0$ & $168.5 \pm 7.6$ & $162.5-177.0$ & $176.1 \pm 5.2$ & $171.0-187.0$ \\
\hline Weight [kg] & $89.6 \pm 21.9$ & $61.7-130.0$ & $70.6 \pm 9.2$ & $61.7-80.0$ & $97.7 \pm 20.8$ & $72.0-130.0$ \\
\hline Body mass index (BMI) & $29.4 \pm 5.7$ & $22.4-37.3$ & $24.8 \pm 2.1$ & $22.4-26.5$ & $31.4 \pm 5.7$ & $22.7-37.3$ \\
\hline Heart rate $[\mathrm{bpm}]$ & $64.0 \pm 9.1$ & $48.0-77.0$ & $55.3 \pm 6.4$ & $48.0-60.0$ & $67.7 \pm 7.6$ & $60.0-77.0$ \\
\hline Systolic blood pressure [mmHg] & $144.5 \pm 13.5$ & $117.0-170.0$ & $142.0 \pm 3.6$ & $139.0-146.0$ & $145.6 \pm 16.3$ & $117.0-170.0$ \\
\hline Diastolic blood pressure [mmHg] & $78.6 \pm 13.7$ & $44.0-95.0$ & $85.3 \pm 3.2$ & $83.0-89.0$ & $75.7 \pm 15.7$ & $44.0-95.0$ \\
\hline Acquisition dates & & & February 2016 - & ember 2016 & & \\
\hline
\end{tabular}

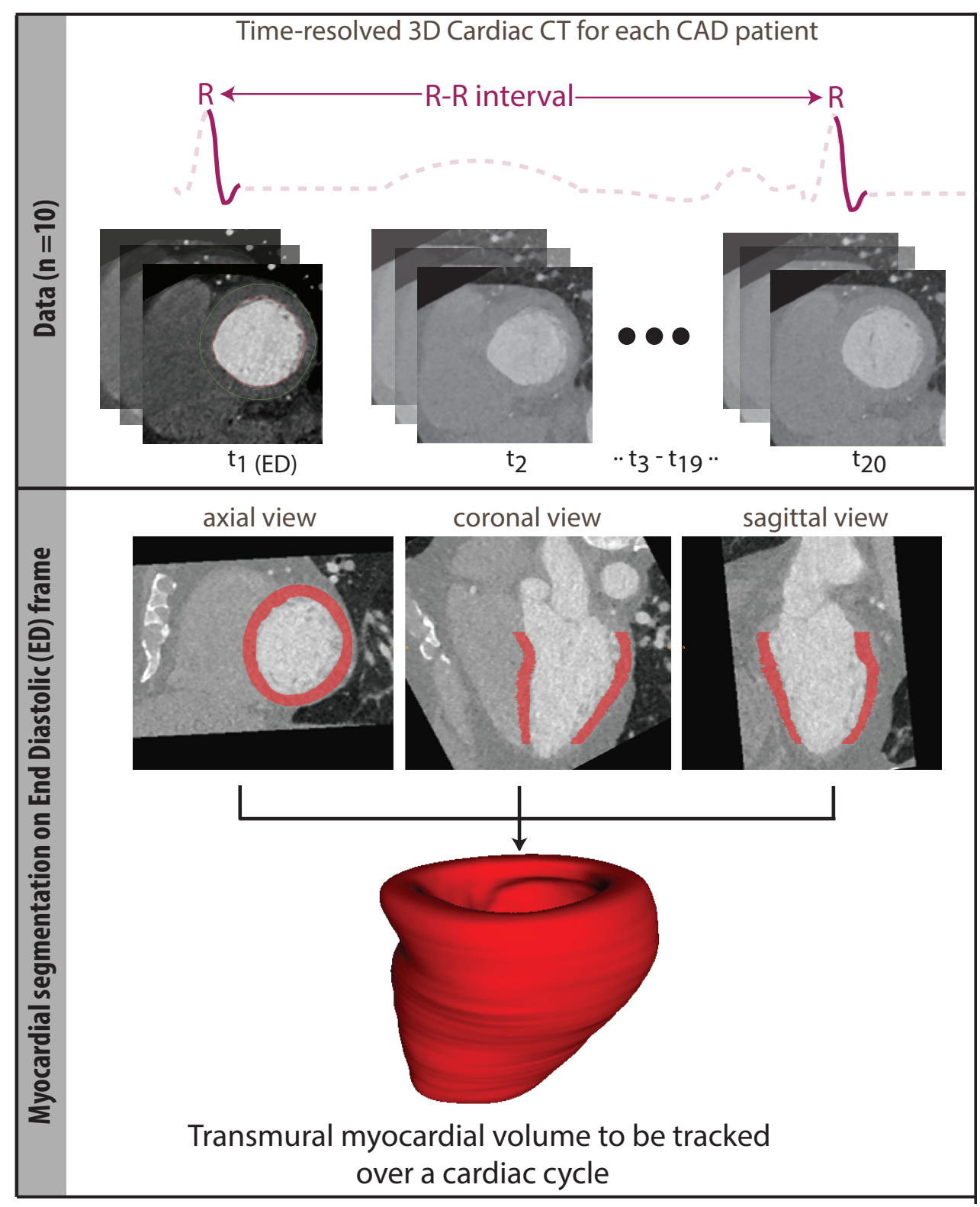

Figure 1: Overview of the materials and segmentation method. The top row shows a typical dataset from one patient comprising $3 \mathrm{D}$ images from multiple phases of a cardiac cycle between two R peaks of an ECG signal. The second row illustrates how the left-ventricular myocardial volume was obtained for an end-diastolic (ED) volume. 


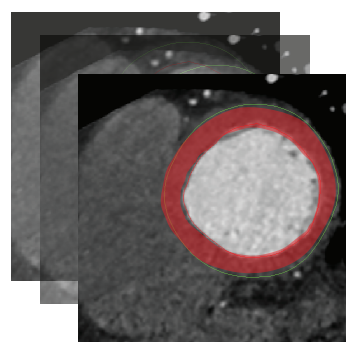

$\mathrm{t}_{1}$ (reference)

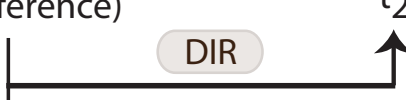

(a) estimation of displacement for each time-frame using DIR

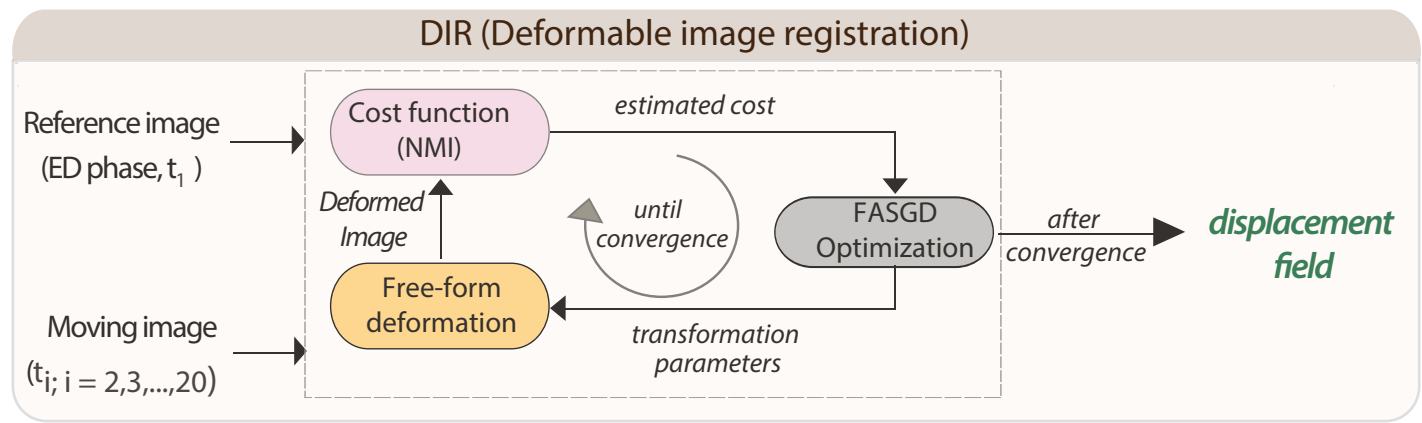

(b) major components of DIR

Figure 2: Myocardial deformation tracking in 3D performed in different cardiac phases with end-diastolic volume as the reference configuration and using a deformable image registration (DIR) strategy as depicted in the second row.

where $I$ is the identity matrix. The Green-Lagrangian strain tensor, $\boldsymbol{E}$, is then estimated using equation 4 .

$$
E=\left(\begin{array}{lll}
E_{x x} & E_{x y} & E_{x z} \\
E_{y x} & E_{y y} & E_{y z} \\
E_{z x} & E_{z y} & E_{z z}
\end{array}\right)=\frac{1}{2}\left(\mathbf{F}^{\mathbf{T}} \mathbf{F}-\mathbf{I}\right) .
$$

$E$ is calculated for myocardial voxels in each cardiac phase and converted to clinically used cylindrical coordinates with circumferential, radial and longitudinal components. The radial vector points to the centroid of the myocardium and the circumferential vector in the counterclockwise tangential direction. The longitudinal direction is from the apex to the basal plane. Segmentwise strain is obtained using the 17 segment model from American Heart Association $(\mathrm{AHA})^{16}$ after identifying the basal, mid-ventricular and apical planes (Figure 3).

Torsion describes the twisting or wringing motion of the myocardium, essentially providing an estimate of the circumferential-longitudinal (CL) shear angle due to counterclockwise rotation in apical tissue and clockwise rotation in basal tissue when viewed from the apex ${ }^{17-19}$. Several definitions exist for torsion estimation, and here we used a version (equation 5 that was shown to provide an unbiased estimate of the 3D torsional angle ${ }^{18 / 19}$.

$$
\text { Torsion }=\frac{\left(\text { rotation }_{\text {apex }} * \text { radius }_{\text {apex }}\right)\left(\text { rotation }_{\text {base }} * \text { radius }_{\text {base }}\right)}{\text { length }_{\text {apex }}-\text { base }_{\text {en }}}
$$

\subsection{Implementation}

DIR and evaluation tools (including statistical tests) were implemented using Elastix ${ }^{12}$ and MATLAB (R2016a, The MathWorks, Inc., USA), respectively, on a workstation with 80 GB RAM, Intel Xeon E5-2620V4/2.1 GHz and NVidia GeForce GTX 1080.

\subsection{Evaluation}

In addition to the myocardial segmentation on the enddiastolic frame for automatic tracking, another ten volumes representing alternate phases of the cardiac cycle were also segmented manually. These are, henceforth, referred to as the ground truth segmentations, required for evaluating the accuracy of the method. 


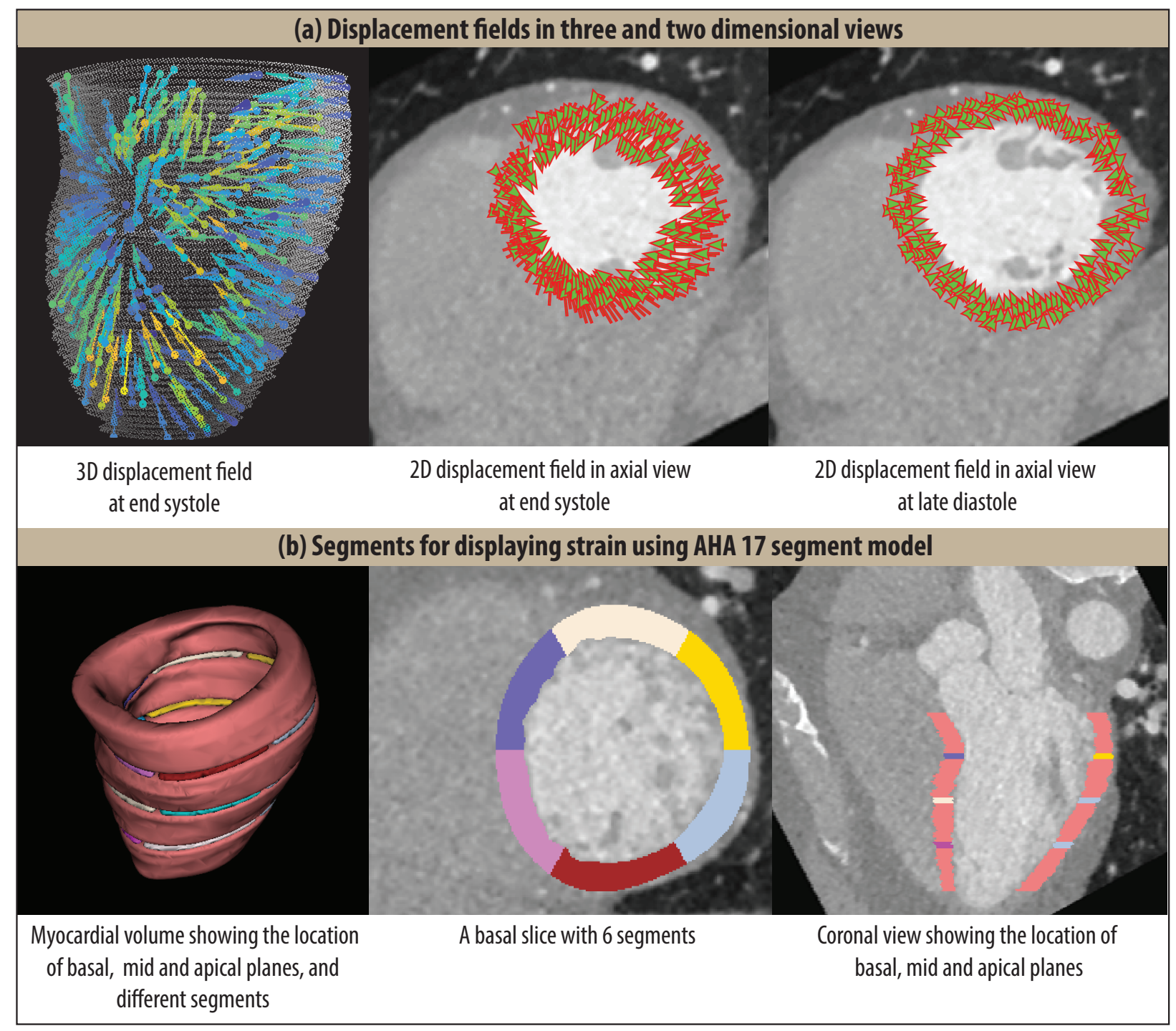

Figure 3: (a) Displacement fields shown using vectors in three and two dimensions at end systole. Also shown are the 2D vectors at late diastole to differentiate between the length of vectors between systole and diastole. These displacement fields are subsampled for the purpose of visualization only. (b) Definition of myocardial segments at different slice levels to represent strain using American Heart Association's (AHA's) 17 segment model

Global accuracy was assessed by measuring the overlap using Dice Similarity Coefficient (DSC) ${ }^{20}$ that is defined as the ratio of intersection between the automatically tracked and ground truth segmentations to their average area. It ranges from 0 to 1 , indicating none to complete overlap. Local accuracy was assessed using point-tocurve (PTC) error. It refers to the shortest distance from each point in the ground truth segmentation to a parametric spline curve defined by closest corresponding points in the automatically tracked myocardium.

In addition, tracking errors $(\delta)$ were estimated to evaluate the efficacy of DIR in bringing the myocardial tissue back to its original position at the end of a cardiac cycle. Defined as the average difference between the original $\left(P_{E D}\right)$ and estimated positions $\left(\hat{P}_{E D}\right)$ of the myocardial voxel positions at end diastole (ED), these errors are cal- culated using equation:

$$
\delta=\frac{1}{N} \sum_{k=1,2, \ldots, N}\left|P_{E D}^{k}-\hat{P}_{E D}^{k}\right|
$$

where $N$ is the total number of myocardial voxels. $\hat{P}_{E D}^{k}$ was obtained by transforming the estimated positions of myocardium at the end of the cardiac cycle back to the reference configuration, using $I_{r}$ as the moving and $I_{m_{20}}$ as the reference image in equation 11. Significance of tracking errors was evaluated using Wilcoxon signed rank test.

To test if the estimated transmural strains and torsion were reliable, only the subjects without any pathological findings in their 4D CT examination could be used for comparison. The 4D CT scans of only three subjects in our co- 


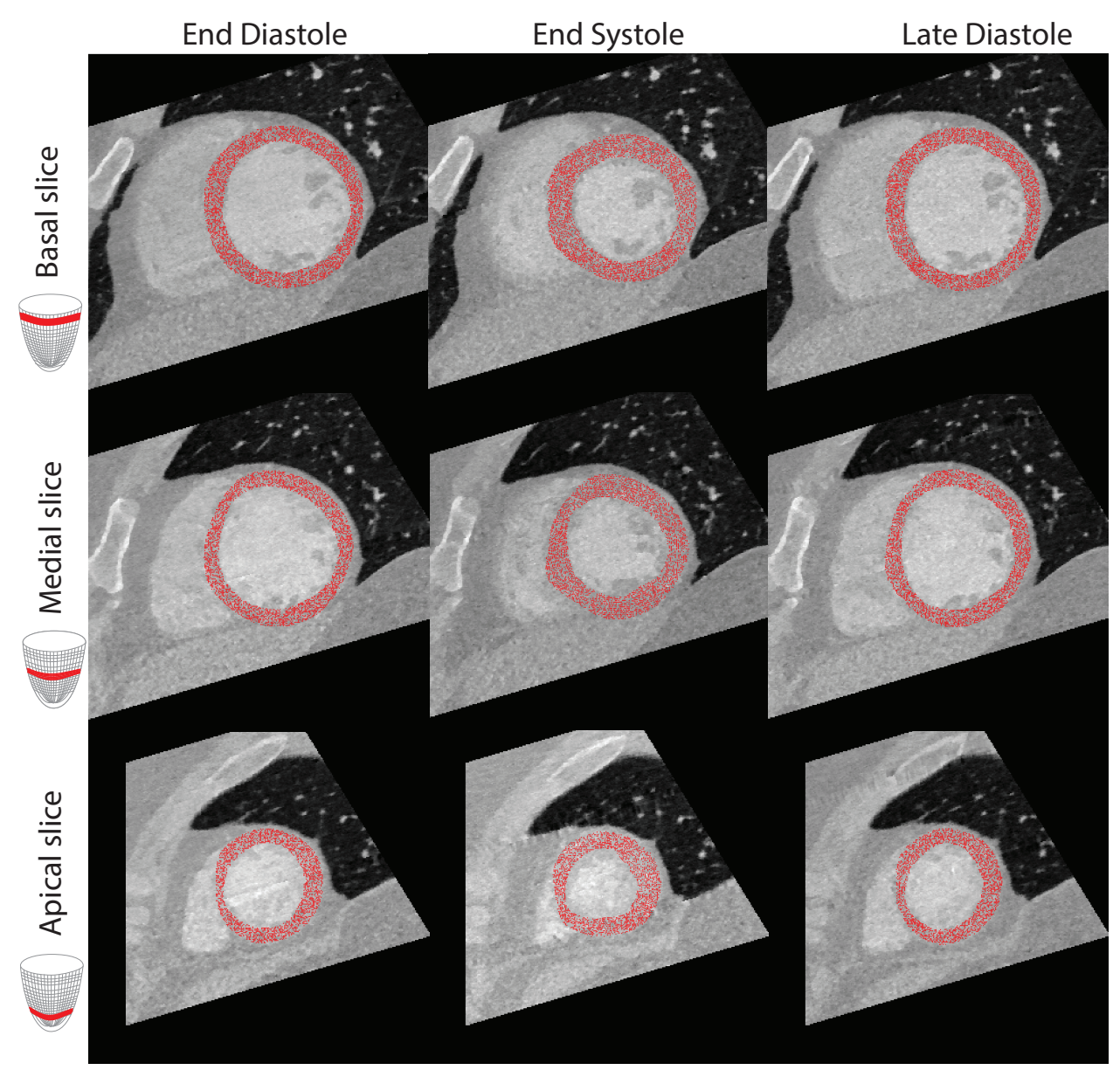

(a) Axial Slices

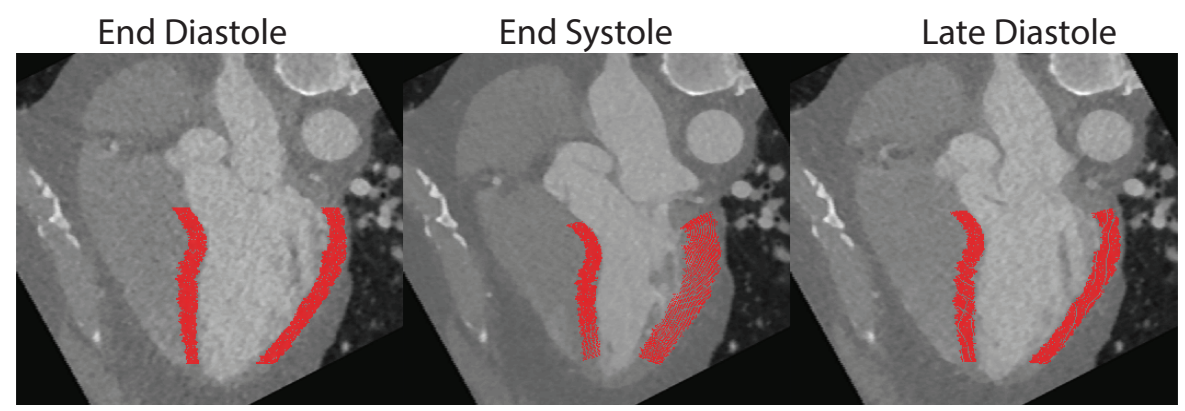

(b) Coronal Slices

Figure 4: Depiction of myocardial volume changes at end systole and late diastole that were automatically obtained by the proposed method.

hort did not show a stenosis, atheromatosis or calcification and a visual assessment of their scans by an experienced cardiologist yielded normal wall motion. These subjects were therefore deemed as normals for strain comparison (Table 2).

\section{Results}

The analysis of all datasets resulted in visually correct myocardial tracking as shown in Figures 3 and 4 ; the former shows the displacement vectors at end systole in three- as well as two-dimensions (2D). The 2D vectors (subsampled for better visibility) are also shown for late diastole resulting in, as expected, smaller displacements in this phase of the cardiac cycle. Due to these displacements, the myocardial tissue moves to different positions, as shown in Figure 4. Of importance is the fact that these displacements are estimated in three dimensions and for each voxel, which is illustrated using multiple views (e.g., axial and coronal). The estimation of these displacements 
Table 2: Outcome of CT examination for each patient specifying the absence/presence in major coronary arteries (LAD: Left Anterior Descending; RCA: Right Coronary Artery; LM: Left Main)

\begin{tabular}{cllc}
\hline Patient \# & \multicolumn{1}{c}{ Diagnosis from 4D CT exam } & Deemed as normal \\
\cline { 1 - 1 } 2 & & Calcified significant stenosis in RCA and LAD & No \\
3 & No pathology (stenosis, atheromatosis, calcification, or wall motion abnormalities) & No \\
4 & General atheromatosis & No \\
5 & Calcification in LAD, no significant stenosis & No \\
6 & Light atherosclerosis in LAD and RCA, no significant stenoses & No \\
7 & General atheromtosis, moderate stenosis in the RCA & No \\
8 & High grade stenosis/occlusion in LAD & Yes & No \\
9 & General atheromatosis, significant stenosis in LM and LAD. & Yes \\
10 & No pathology (stenosis, atheromatosis, calcification, or wall motion abnormalities) & \\
\hline
\end{tabular}

over an entire cardiac cycle took approximately 35 minutes per patient (compared to up to 900 minutes in Peyrat et al. 4 ).

Geometric accuracy (mean \pm std) was evaluated using DSC $(0.99 \pm 0.05)$ and PTC $(0.56 \mathrm{~mm} \pm 0.47 \mathrm{~mm})$ for the entire cohort. The baseline/reference for these measures were the ground truth segmentations described in section 2.3. Temporal variations in DSC and PTC (Figure 50 measurements show low-to-negligible variation even with high noise levels, especially, in later parts of the cardiac cycle (70-95\%). The box plots in Figure 5 show the percentiles of DSC and PTC across ten frames for each patient.

Tracking errors are shown in Figure 6for different slices. Per patient, these errors were measured for the entire myocardial volume and subsequently averaged according to the different slice levels to identify regional differences. Averaged over all the patients, these errors were $0.13 \pm 0.09 \mathrm{~mm}, 0.64 \pm 0.46 \mathrm{deg}$ and $0.10 \pm 0.05 \mathrm{~mm}$ in radial, angular and longitudinal directions, respectively. Wilcoxon signed ranked test showed insignificant $(p>0.9$ ) differences in the positions of original and estimated points.

Conservation of volume is an important characteristic of myocardial wall as it deforms over a cardiac cycle. It is depicted for each patient and for each phase using the determinant of Jacobian $(\operatorname{det}(J))$ in Figure $7 \mathrm{~A}$; $\operatorname{det}(J)>0$ indicates realistic deformations and $\operatorname{det}(J) \approx 1$ for negligible volume change.

Global and mid-ventricular only strain estimates for three subjects with normal myocardial function (Table 2) were compared with the reported ranges using MRI based feature tracking (MRI-FT) 21 and MRI based tagging ${ }^{22}$ in Table 3. Variation of different strain components over a cardiac cycle for these subjects is shown in Figure $7 \mathrm{~B}$. These temporal variations reflect the normal contraction of myocardium. Also shown in Table 3 is the comparison of average peak systolic torsion estimates $\left(7.22^{\circ} \pm 2.3^{\circ}\right)$ for the same subjects and the reported torsion values for normals $\left(7.7^{\circ} \pm 1.4^{\circ}\right)$ from MRI based tagging 18 .

Figure 8 shows the feasibility of the proposed method in assisting clinical diagnoses using an example of a patient with significant stenosis in left anterior descending (LAD) artery. Segmentwise strain in circumferential, radial and longitudinal directions are shown using the $\mathrm{AHA}$ model ${ }^{16}$. Also shown are voxelwise transmural circumferential strain in different views for the same patient as the circumferential strain has been investigated more widely for patients suspected of CAD 23.25 .

\section{Discussion}

We presented a deformable image registration based tracking approach to quantify regional myocardial motion in 4D CT over an entire cardiac cycle and showed its potential for patient specific in vivo measurement of transmural myocardial strain. With a high accuracy on clinical datasets as opposed to only synthetic and canine data ${ }^{45}$, and relatively lower computation time of 35 mins (as opposed to up to 900 minutes in ${ }^{4}$ ), the proposed method enables automatic tracking of the desired myocardial volume without incurring additional costs to clinicians.

Geometric accuracy evaluation, taking into account both the global shape (DSC) and finer details (PTC) of the expected anatomy, allowed for a robust assessment of the quality of deformation tracking. Compared to Tavakoi et al. ${ }^{2}$, where the manual vs automatic average point differences were higher than $2.9 \mathrm{~mm}$, the corresponding average errors (PTC) in the current approach were lower than $0.6 \mathrm{~mm}$. Notably, consistently high overlap ratio and low PTC errors were achieved despite large variations in patient size, cardiac motion due to varying degree of coronary stenosis, and signal-to-noise ratio caused by dose modulation. Furthermore, low tracking errors for different slices and segments illustrate the ability of the proposed method in bringing the myocardium back to its original configuration. Since the B-spline based transformation model allows for sub-voxel displacements, the scale of tracking errors was not limited only to voxels as shown 

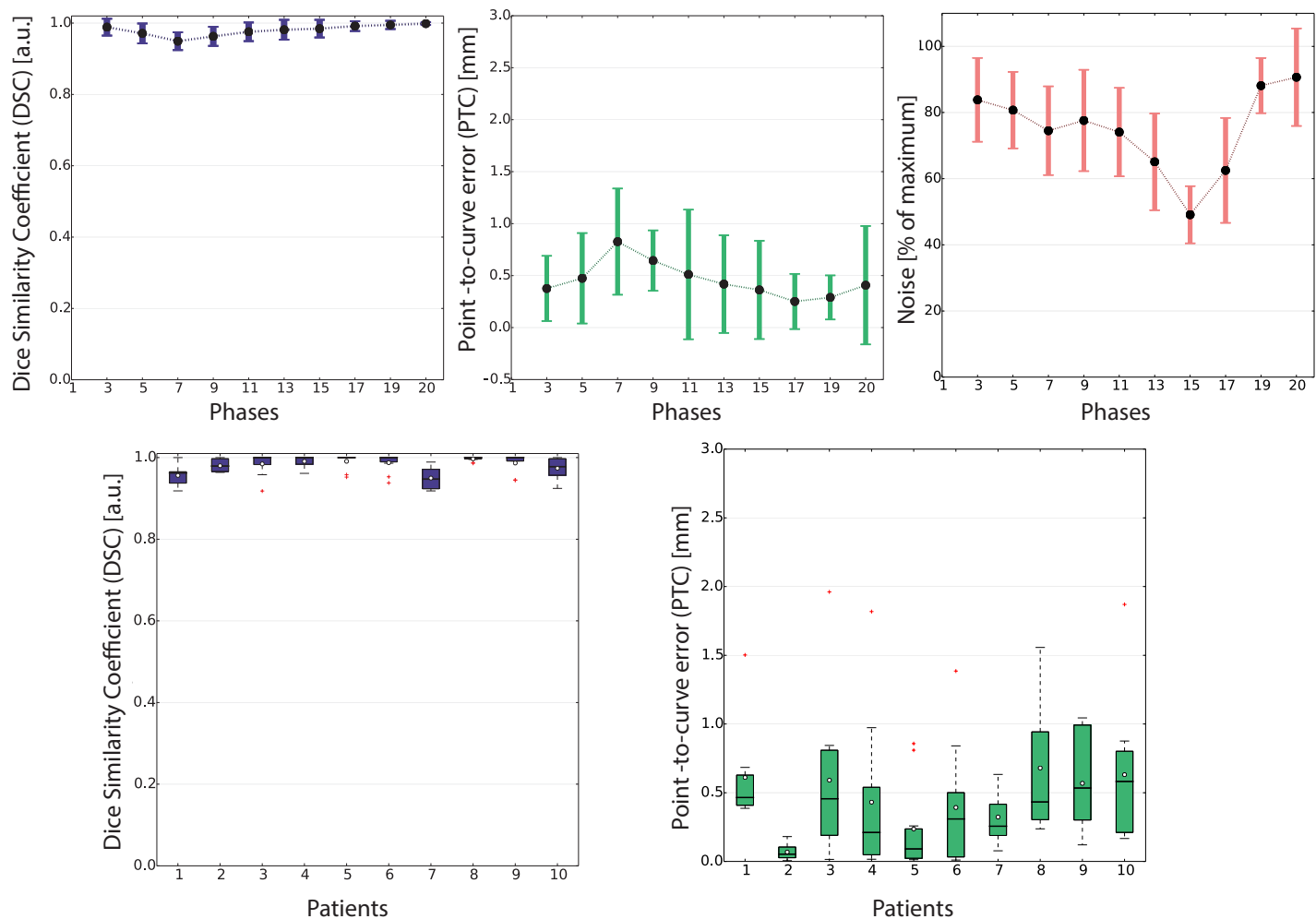

Figure 5: Demonstration of geometric accuracy using Dice similarity coefficient (DSC) and point-to-curve (PTC) error between the ground truth and automatically tracked left-ventricular myocardial geometry. The mean and standard deviations in DSC, PTC, and noise across the entire cohort are shown for each phase of the cardiac cycle in the top row. The box plots in the bottom row show the medians, means (white circles), interquartile ranges, and outliers across all evaluated phases for each patient.

Table 3: Comparison of estimated strain (\%) and average peak systolic torsion (CL shear angle in degrees) from CT for patients with normal function to the reported values in the literature from MRI

\begin{tabular}{|c|c|c|c|c|c|}
\hline \multicolumn{6}{|c|}{ END SYSTOLIC STRAIN (\%) } \\
\hline & \multicolumn{2}{|c|}{ Mid-ventricular only } & \multicolumn{3}{|c|}{ Global } \\
\hline & CT & MRI-FT21 & CT & MRI-FT21 & MRl-tagging 22 \\
\hline Circumferential & -20 & {$[25.9,-21.3]$} & -23.5 & {$[-24.2,-21.1]$} & {$[-20,15]$} \\
\hline Radial & 28.3 & {$[23.6,32.4]$} & 31.2 & {$[29.2,49.0]$} & {$[30,40]$} \\
\hline Longitudinal & -18.3 & Not available & -17.2 & {$[-21.0,-17.8]$} & {$[-20,-15]$} \\
\hline \multicolumn{6}{|c|}{ PEAK SYSTOLIC TORSION $\left({ }^{\circ}\right)$} \\
\hline & \multicolumn{2}{|r|}{ CT } & \multicolumn{3}{|c|}{ MRI-tagging ${ }^{17}$} \\
\hline & \multicolumn{2}{|c|}{$7.2 \pm 2.3$} & \multicolumn{3}{|c|}{$7.7 \pm 1.4$} \\
\hline
\end{tabular}

by the average of less than $0.5 \mathrm{~mm}$, which is the size of a voxel in this study.

Free-form deformation models such as the one used in this study can be susceptible to unnatural wraps and additional regularization schemes are often required to counter such problems. However, implicit regularization in B-spline based transformation helps in avoiding implausible foldings and holes by penalizing the discontinuities in non-rigid transformation. As evident from the non-zero $\operatorname{det}(J)$, it is indeed the case here. The volumes of the deformed images were also conserved across all the patients as shown by $\operatorname{det}(J) \approx 1$. In addition, by employing a meshless strategy as opposed to the mesh-based biomechanical model as proposed in Lamash et al. ${ }^{1}$, setting of multiple model parameters can be precluded.

Reliability assessment of estimated strain from CT alone is challenging, especially, in the pathological cases where no consensus exists regarding the expected strain values. However, several studies have been performed in healthy subjects and the normal values show a considerable over- 

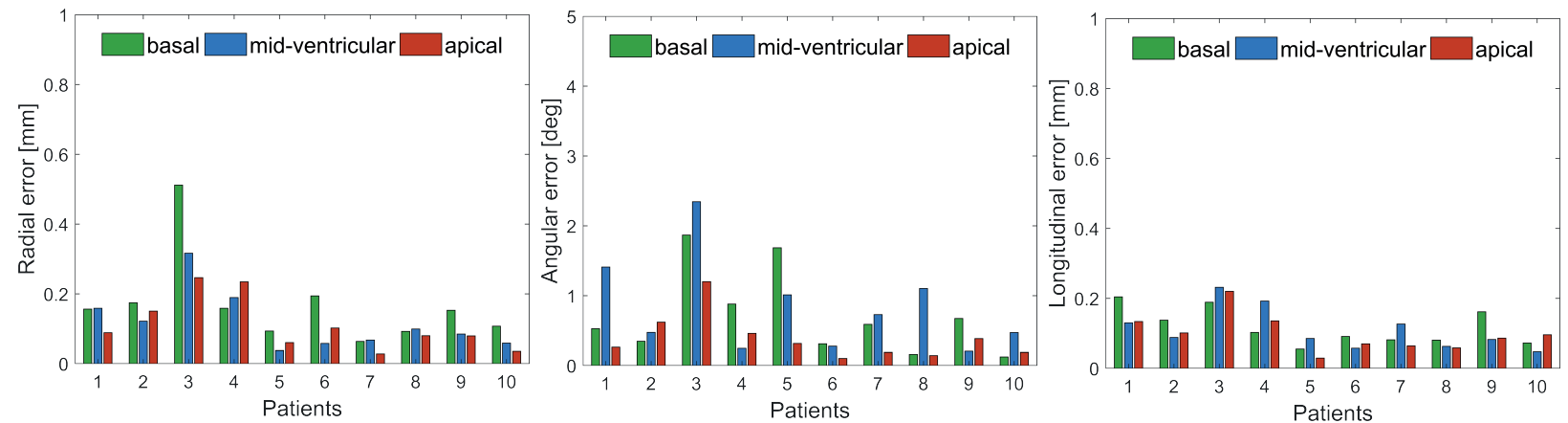

(a) Tracking errors for each patient at different slice levels in the left ventricle
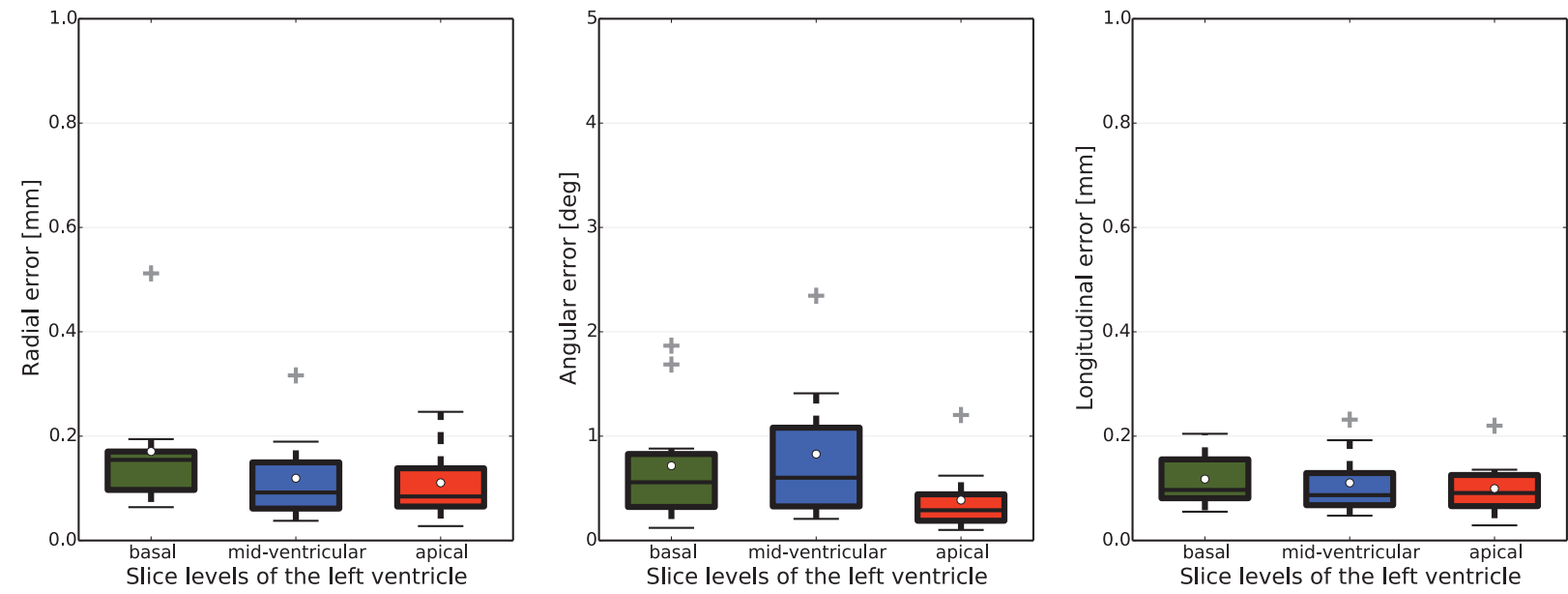

(b) Tracking errors for the entire cohort at different slice levels in the left ventricle

Figure 6: Tracking error in radial, angular and longitudinal directions shown in: (a) for each patient at different levels in the left ventricle and (b) for the entire cohort at different slice levels.

lap between multiple studies. Hence, without the data from other modalities, a reasonable assessment of the validity of estimated strains is to compare the normal values with the existing literature. To this end, only those subjects in our cohort could be considered for comparing with the existing studies for whom no abnormalities (e.g., stenosis, atherosclerosis, etc.) were shown on the clinical 4D CT examinations; only three such subjects could be identified in our cohort (Table 2). Our strain estimates for those subjects (circumferential: $-23.5 \%$, radial: $31.2 \%$, longitudinal: $-17.2 \%$ ) were within the range of reported strain distributions (circumferential: [-24.2\%, $-15 \%]$, radial: [30.0\%, 49.0\%], longitudinal: [-21\%, $-15 \%]$ ) in MRI based feature-tracking $(\mathrm{FT})^{21}$ and tagging ${ }^{22}$. Average torsion estimate from the proposed method $\left(7.22^{\circ}\right)$ follows a similar pattern i.e., it was in conformity with the reported value $\left(7.7^{\circ}\right)^{18}$. Unlike strain, however, it has not been reported as extensively for normals and the available studies employ many different definitions ${ }^{19}$, thus limiting the ability for a wider comparison.

The reported estimates of left ventricular strain typically come either from MRI or US and both of them have lower spatial resolution than CT. As such, low resolution restricts the assessment to coarsely distributed locations in the myocardium. A high-resolution CT providing similar strain estimates at global and segmental level, therefore, has the ability for even finer visualization of myocardial strain, an example of which is shown in Figure 8. The possibility of selecting any voxel, region, or the entire myocardium for displacement and strain visualization may potentially help clinicians in characterizing myocardial motion at much finer detail than is possible with existing modalities.

\section{Limitations}

Although registration, and hence, the tracking itself is unsupervised, left ventricular segmentation at a reference phase is required to identify the region of interest to be tracked i.e., the myocardium. Commercial segmentation tools from major vendors can be used for this purpose. In addition, the evaluation was performed on a small cohort including 10 subjects and shows only the proof-of-concept for CT-based tracking and subsequent strain estimation. However, the encouraging results should lead to a more extensive evaluation of the method for detecting myocardial wall motion abnormalities. 

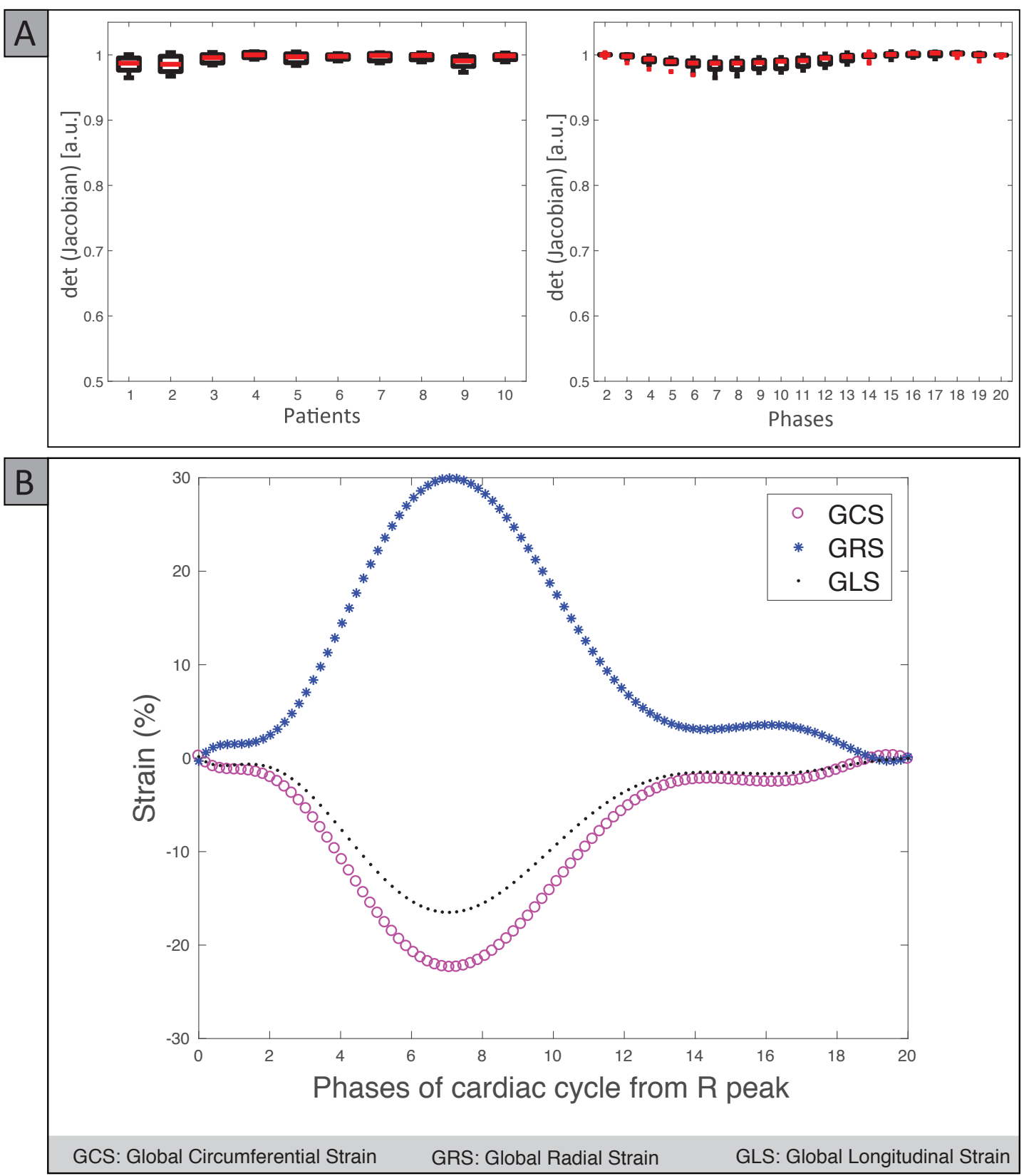

Figure 7: Illustration of change in myocardial volume and strain over a cardiac cycle. (A) Variation in determinant of Jacobian (det(J)) for each patient and phase of the cardiac cycle. For invertible and realistic deformation $\operatorname{det}(\mathrm{J})>0 . \operatorname{det}(\mathrm{J}) \approx 1$ across the cardiac cycle confirms conservation of myocardial volume despite the variation in strain components. (B) Variation in global strain components (GCS: Global Circumferential Strain, GRS: Global Radial Strain, and Global Longitudinal Strain) through a cardiac cycle averaged for subjects with normal myocardial function.

\section{Future applications}

Quantification of myocardial deformation allows for early detection of $C A D^{26}-29$ as well as treatment guidance by detecting myocardial regions with abnormal contraction. Cardiac CT based quantification of such motion would thus serve as an excellent complement to coronary stenosis detection. In addition to left ventricular strain estimation, the proposed method may also facilitate the assessment of pulsatile blood flow from the cardiac chambers into the coronaries and other major vessels as shown by Lantz et al. .30 .

\section{Conclusion}

The proposed method provides an automatic and robust tracking of left ventricular myocardium in time-resolved cardiac CT images over an entire cardiac cycle. Our results demonstrate its potential in enabling a reliable assessment of diagnostically useful functional information 


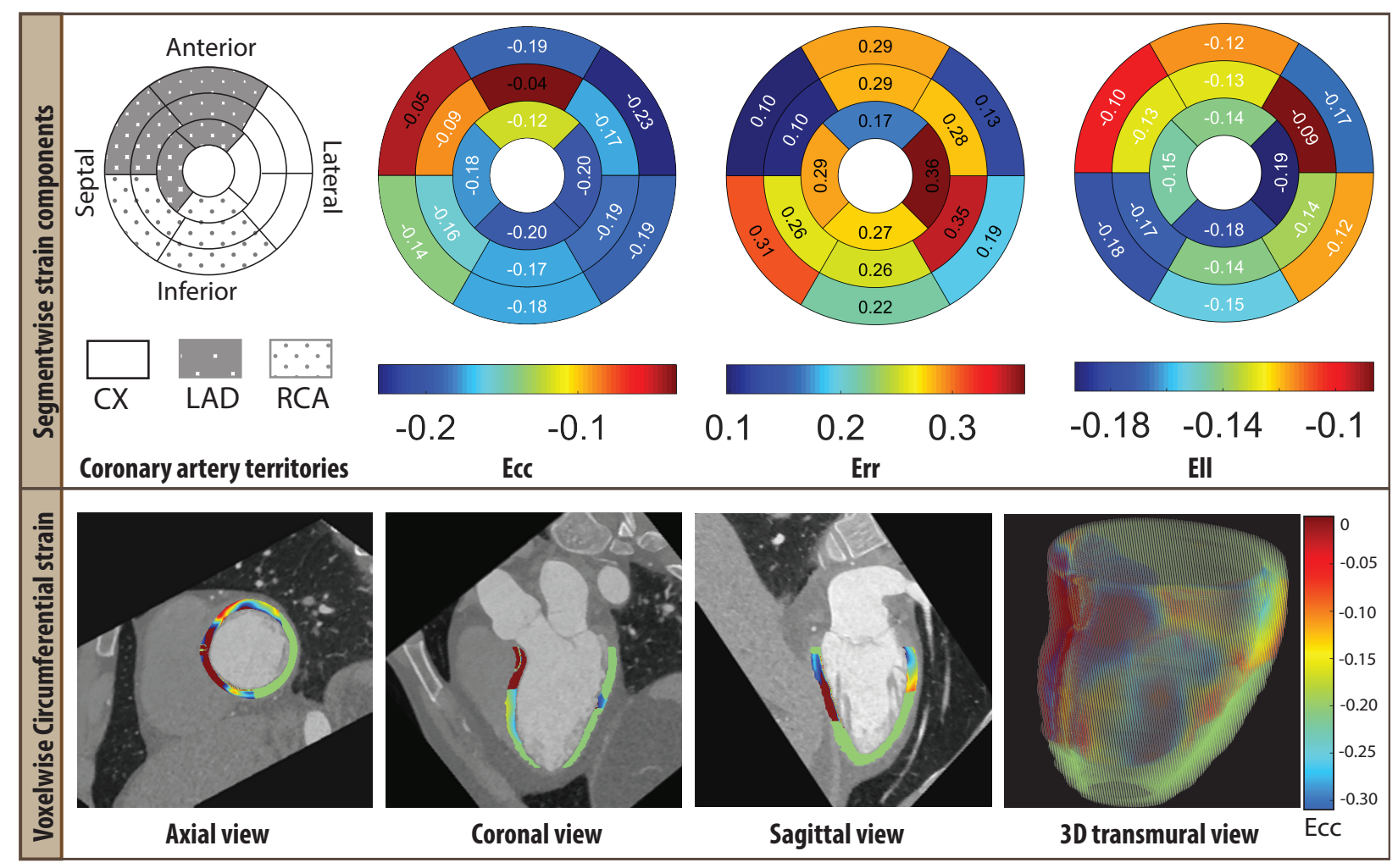

Figure 8: Segmentwise circumferential $\left(E_{c c}\right)$, radial $\left(E_{r r}\right)$ and longitudinal $\left(E_{l l}\right)$ strain at end systole for a patient with significant stenosis in left anterior descending (LAD) coronary artery. Circumferential strain is also shown voxelwise for the same patient at end systole. Corresponding segments in the AHA model as well as the regions in voxelwise representation are shown to have lower strain compared to the rest of the myocardium. The 3D voxelwise representation has been shown with downsampled data for better visibility and transparency.

on wall motion from time-resolved cardiac CT images that are routinely acquired in clinics worldwide.

\section{Acknowledgements}

This work was funded by Knut and Alice Wallenberg foundation (grant: KAW 2013.0076).

\section{Disclosure}

The authors have nothing to disclose.

\section{References}

1. Lamash $Y$, Fischer $A$, Carasso $S$, et al. Strain analysis from 4-D cardiac CT image data. IEEE Trans Biomed Eng. 2015;62(2):511521.

2. Tavakoli V and Sahba N. Cardiac motion and strain detection using 4D CT images: comparison with tagged MRI, and echocardiography. Int J Cardiovasc Imaging. 2014;30(1):175-184.

3. Pourmorteza A, Chen MY, van derPals J, et al. Correlation of CTbased regional cardiac function (SQUEEZ) with myocardial strain calculated from tagged MRI: an experimental study. Int J Cardiovasc Imaging. 2016;32(5):817-823.

4. Peyrat JM, Delingette H, Sermesant M, et al. Registration of 4D cardiac CT sequences under trajectory constraints with multichannel diffeomorphic demons. IEEE Trans Med Imaging. 2010;29(7):13511368.
5. Wong KC, Tee M, Chen M, et al. Myocardial strain estimation from CT: towards computer-aided diagnosis on infarction identification. In SPIE Med Imaging. 2015; 941434-941434.

6. Wong KC, Tee M, Chen M, et al. Regional infarction identification from cardiac CT images: a computer-aided biomechanical approach. Int J Comput Assisted Radiol Surg. 2016;11(9):1573-1583.

7. Jakobs TF, Becker CR, Ohnesorge B, et al. Multislice helical CT of the heart with retrospective ECG gating: reduction of radiation exposure by ECG-controlled tube current modulation. Eur Radiol. 2002;12(5):1081-1086.

8. Heiberg E, Sjögren J, Ugander M, et al. Design and validation of Segment - freely available software for cardiovascular image analysis. BMC Med Imaging. 2010;10(1):1.

9. Tee M, Noble JA, and Bluemke DA. Imaging techniques for cardiac strain and deformation: comparison of echocardiography, cardiac magnetic resonance and cardiac computed tomography. Expert Rev Cardiovasc Ther. 2013;11(2):221-231.

10. Rueckert D, Sonoda LI, Hayes C, et al. Nonrigid registration using free-form deformations: application to breast MR images. IEEE Trans Med Imaging. 1999;18(8):712-721.

11. Szeliski $R$ and Lavallée $S$. Matching 3-D anatomical surfaces with non-rigid deformations using octree-splines. Int J Comput Vision. 1996;18(2):171-186.

12. Klein S, Staring M, Murphy K, et al. Elastix: a toolbox for intensitybased medical image registration. IEEE Trans Med Imaging. 2010; 29(1):196-205.

13. Qiao $Y$, vanLew $B$, Lelieveldt BP, et al. Fast automatic step size estimation for gradient descent optimization of image registration. IEEE Trans Med Imaging. 2016;35(2):391-403.

14. Collignon A, Maes F, Delaere D, et al. Automated multi-modality image registration based on information theory. In Inf Process Med 
Imaging., volume 3. 1995; 263-274.

15. Suever JD, Wehner GJ, Jing L, et al. Right ventricular strain, torsion, and dyssynchrony in healthy subjects using 3D spiral cine DENSE magnetic resonance imaging. IEEE Trans Med Imaging. 2017;36(5):1076-1085.

16. Cerqueira MD. American Heart Association Writing Group on Myocardial Segmentation and Registration for Cardiac Imaging: Standardized myocardial segmentation and nomenclature for tomographic imaging of the heart: a statement for healthcare professionals from the Cardiac Imaging Committee of the Council on Clinical Cardiology of the American Heart Association. Circulation. 2002; 105:539-542.

17. Rüssel IK, Götte MJ, Kuijer JP, et al. Regional assessment of left ventricular torsion by CMR tagging. J Cardiovasc Magn Reson. 2008;10(1):26.

18. Rüssel IK, Marcus JT, Kuijer JP, et al. Comparison of 2D and 3D calculation of left ventricular torsion as circumferential-longitudinal shear angle using cardiovascular magnetic resonance tagging. $J$ Cardiovasc Magn Reson. 2009;11(1):8.

19. Young $A A$ and Cowan BR. Evaluation of left ventricular torsion by cardiovascular magnetic resonance. J Cardiovasc Magn Reson. 2012;14(1):49.

20. Dice LR. Measures of the amount of ecologic association between species. Ecology. 1945;26(3):297-302.

21. Vo HQ, Marwick TH, and Negishi K. MRI-Derived Myocardial Strain Measures in Normal Subjects. JACC Cardiovasc Imaging. 2017; 2245.

22. Jeung MY, Germain P, Croisille P, et al. Myocardial tagging with MR imaging: overview of normal and pathologic findings. Radiographics. 2012;32(5):1381-1398.

23. Kar J, Knutsen AK, Cupps BP, et al. A validation of two-dimensional In Vivo regional strain computed from displacement encoding with stimulated echoes (DENSE), in reference to tagged magnetic resonance imaging and studies in repeatability. Annals of Biomed Eng. 2014;42(3):541-554.

24. Miyagi $\mathrm{H}$, Nagata M, Kitagawa $\mathrm{K}$, et al. Quantitative assessment of myocardial strain with displacement encoding with stimulated echoes MRI in patients with coronary artery disease. Int $\mathrm{J}$ Cardiovasc Imaging. 2013;29(8):1779-1786.

25. Kihlberg J, Haraldsson $H$, Sigfridsson A, et al. Clinical experience of strain imaging using DENSE for detecting infarcted cardiac segments. J Cardiovasc Magn Reson. 2015;17(1):50.

26. Jiang $\mathrm{K}$ and $\mathrm{Yu} X$. Quantification of regional myocardial wall motion by cardiovascular magnetic resonance. Quant Imaging Med Surg. 2014;4(5):345.

27. Wang $\mathrm{H}$ and Amini $\mathrm{AA}$. Cardiac motion and deformation recovery from MRI: a review. IEEE Trans Med Imaging. 2012;31(2):487-503.

28. Götte MJ, vanRossum AC, Twisk JW, et al. Quantification of regional contractile function after infarction: strain analysis superior to wall thickening analysis in discriminating infarct from remote myocardium. J Am Coll Cardiol. 2001;37(3):808-817.

29. Marcus JT, Götte MJ, Van Rossum AC, et al. Myocardial function in infarcted and remote regions early after infarction in man: assessment by magnetic resonance tagging and strain analysis. Magn Reson Med. 1997;38(5):803-810.

30. Lantz J, Henriksson L, Persson A, et al. Patient-specific simulation of cardiac blood flow from high-resolution computed tomography. $J$ Biomech Eng. 2016;138(12):121004-9. 